\title{
Precoded Distributed Space-Time Block Codes in Cooperative Diversity-Based Downlink
}

\author{
Hilde Skjevling, Student Member, IEEE, David Gesbert, Senior Member, IEEE, \\ and Are Hjørungnes, Senior Member, IEEE
}

\begin{abstract}
Cooperative diversity is a rapidly emerging topic for wireless communications, with ad hoc and hybrid/relay networks as two main applications so far. In this paper, we investigate the cooperative diversity concept for MIMO multicell networks, where the processing must be optimized to account for the variability of the channel conditions across the cooperative devices. This can be done via distributed precoding and is realistically based on channel statistics (average gains, correlations, etc.). We give a new approach to the previously coined equal diversity spread principle, through minimization of an approximated SER expression. Next, we focus on a low-complexity approach to minimizing a PEP-based performance measure. Gains are evaluated in a multicell scenario with collaborating base stations.
\end{abstract}

Index Terms - Cooperative systems, diversity methods, minimum symbol error rate (SER), orthogonal space-time block code (OSTBC), precoder.

\section{INTRODUCTION}

$\mathbf{M}$ ULTIPLE-Input Multiple-Output (MIMO) signal processing promises substantial increase in wireless network capacity, through the well-defined multiplexing and diversity gains [1]-[3]. One way to provide spatial diversity at both ends of the communication link, is the use of Orthogonal Space-Time Block Codes (OSTBC) [4], and a large body of work has been dedicated to precoding and power allocation for point-to-point MIMO communication systems, see e.g. [5]-[7].

MIMO systems yield their best for uncorrelated channel matrix elements, so recent interest focuses on network scenarios providing additional and independent sources of diversity. These typically include setups where the multiple antenna elements are distributed over the network, instead of being localized on a single device. Prominent examples are (i) the multiuser multicell MIMO setup, where one or more access points address the data needs of multiple user terminals simultaneously and in a joint fashion [8]-[10], and by (ii) the

Manuscript received December 9, 2005; revised January 21, 2007; accepted July 28, 2007. The associate editor coordinating the review of this letter and approving it for publication was M. Uysal. This work was supported by the Research Council of Norway ( $\mathrm{RCN}$ ) and the French Ministry of Foreign Affairs, through the Aurora project "Optimization of Broadband Wireless Communications Networks" and RCN project 160637.

H. Skjevling is with the Department of Information, University of Oslo, PO Box 1080, Blindern, 0316 Oslo, Norway (e-mail: hildesk@ifi.uio.no).

D. Gesbert is with the Mobile Communications Department, Eurécom Institute, BP 193, F-06904 Sophia Antipolis Cédex, France (e-mail: gesbert@eurecom.fr).

A. Hjørungnes is with UniK-University Graduate Center, University of Oslo, PO Box 70, 2027 Kjeller, Norway (e-mail: arehj@unik.no).

Digital Object Identifier 10.1109/TWC.2007.051008. cooperative diversity setup, where multiple devices collaborate to combat the detrimental effects of fading [11].

In many cooperative scenarios, single-antenna user terminals function as relays, to help a source terminal reach a target destination [12]-[17]. Thus, the relaying phase serves as a trick to broadcast the coded symbols over the spatially scattered transmitters, which then perform distributed processing towards a common destination. Cooperative diversity schemes notably include the use of Space-Time Block Codes (STBC), and the spatial elements of the codewords are distributed over the antennas of the collaborating devices [13], [17]-[20].

In this paper, we focus on the cooperative processing aspect, using a distributed OSTBC, ignoring issues associated with the relay protocol. A relevant example is the downlink cooperative diversity in a multicell network, where inter-connected base stations (BS) collaborate to serve one mobile user (MU), and all participants may be equipped with multiple antennas. Each BS owns a copy of the codeword intended to the MU. The results apply to terminal-based relay networks as well. Given the large-scale separation of the transmitters, the channel conditions from each BS to the common MU may be different, unlike the scenario of point-to-point MIMO.

The cooperative diversity scheme should be optimized with respect to the channel conditions. We tackle this challenge via distributed precoding, which we optimize based on channel statistics, rather than on the more overhead-incurring instantaneous channel knowledge. We investigate low complexity approximated algorithms for optimization of the precoder, with the following main contributions: 1) We generalize the equal diversity spread principle, first presented in [21], to multiple transmit antennas, and provide a justification behind it in the form of a Gaussian approximation that simplifies the exact Symbol-Error-Rate (SER) expression. 2) We present an optimization problem and an iterative solution based on minimizing the Pairwise Error-Probability (PEP). We evaluate our solutions numerically for a multicell network, where several BSs cooperate to serve a single MU.

This paper is organized as follows: Section II describes the system model, and Section III presents the minimum SER precoding problem. Section IV gives some approaches to reduced complexity precoder optimization. We present our simulation results in Section V, and conclude in Section VI.

\section{Signal And Channel Model}

We consider $L$ spatially distributed base stations, with $M_{t_{l}}$ antennas each, engaged in cooperative downlink communication towards one mobile unit, with $M_{r}$ antennas. The 
distributed MIMO-system has a total of $M_{t}=\sum_{l=0}^{L-1} M_{t_{l}}$ transmit and $M_{r}$ receive antennas.

The transmit correlation is assumed negligible, thanks to generous transmit antenna and inter-cell spacing. Terminal antennas will be correlated, however. The degree of receive correlation, as well as the path loss and slow fading coefficients, are assumed to depend on which BS the signal originates from. The average channel gains can be equalized, using slow power control. We consider both equal and unequal average signal-to-noise ratio (SNR). The BSs are assumed synchronized upon transmission, via fast links to a central unit. The channel is flat fading, but results are expected to carry over to an OFDM setting.

The $L$ BSs collectively transmit a vector of independent symbols $\boldsymbol{x}=\left[x_{0}, x_{1}, \ldots, x_{K-1}\right]^{T}$, obtained from $M K$ bits, using $M$-ary, linear and memoryless modulation. Among the possible cooperation schemes, we choose the cooperative diversity setup, applying an OSTBC to the vector $\boldsymbol{x}$, yielding the $B \times N$ matrix $\boldsymbol{C}(\boldsymbol{x})$. To compensate for the effect of uneven path loss, we apply linear precoding, given by the $M_{t} \times B$ matrix $\boldsymbol{F}$, before launching the codeword. The $M_{r} \times N$ receive matrix at the MU builds as $\boldsymbol{Y}=\boldsymbol{H} \boldsymbol{F} \boldsymbol{C}(\boldsymbol{x})+\boldsymbol{V}$, where the $M_{r} \times N$-sized matrix $\boldsymbol{V}$ is the additive, white noise, $v_{i j} \sim \mathcal{C N}\left(0, \sigma_{v}^{2}\right)$. After reception, Maximum Likelihood (ML) decoding, and demodulation is performed. A model of the chain is shown in Fig. 1.

The overall $M_{r} \times M_{t}$-sized MIMO channel is $\boldsymbol{H}=\boldsymbol{H}_{c} \boldsymbol{P}$, where $\boldsymbol{H}_{c}=\left[\begin{array}{llll}\boldsymbol{h}_{c_{0}} & \boldsymbol{h}_{c_{1}} & \cdots & \boldsymbol{h}_{c_{M_{t}-1}}\end{array}\right]$ represents correlation and fast fading, while $\boldsymbol{P}=\operatorname{diag}\left(\left[\sqrt{\rho_{0}}, \sqrt{\rho_{1}}, \cdots, \sqrt{\rho_{M_{t}-1}}\right]\right)$ gives the path loss. If antennas $i$ and $k$ are located on the same BS, then $\rho_{i}=\rho_{k}$. Further decomposition gives $\operatorname{vec}\left(\boldsymbol{H}_{c}\right)=$ $\boldsymbol{R}^{1 / 2} \operatorname{vec}\left(\boldsymbol{H}_{w}\right)$, where $\boldsymbol{R}$ is the global correlation matrix and $\boldsymbol{H}_{w} \in \mathcal{C}^{M_{r} \times M_{t}}$ contains the fast fading coefficients, $\left(\boldsymbol{H}_{w}\right)_{i, j}=h_{w_{i_{j}}} \sim \mathcal{C N}(0,1)$. The vec-operator stacks the columns of a matrix into a vector, from left to right.

$$
\begin{aligned}
\boldsymbol{R} & =\mathbb{E}\left[\operatorname{vec}\left(\boldsymbol{H}_{\boldsymbol{c}}\right) \operatorname{vec}^{H}\left(\boldsymbol{H}_{\boldsymbol{c}}\right)\right] \\
& =\left[\begin{array}{cccc}
\boldsymbol{R}_{r_{0}} & 0_{M_{r} \times M_{r}} & \ldots & \mathbf{0}_{M_{r} \times M_{r}} \\
\mathbf{0}_{M_{r} \times M_{r}} & \boldsymbol{R}_{r_{1}} & \ldots & \mathbf{0}_{M_{r} \times M_{r}} \\
\vdots & \vdots & \ddots & \vdots \\
\mathbf{0}_{M_{r} \times M_{r}} & \mathbf{0}_{M_{r} \times M_{r}} & \ldots & \boldsymbol{R}_{r_{M_{t}}}
\end{array}\right] .
\end{aligned}
$$

$\boldsymbol{R}_{i}=\mathbb{E}\left[\boldsymbol{h}_{c_{i}} \boldsymbol{h}_{c_{i}}^{H}\right]$ is the correlation of the $i$-th transmit path. We have $\boldsymbol{H}=\left[\begin{array}{lllll}\boldsymbol{R}_{r_{0}}^{1 / 2} \boldsymbol{h}_{w_{0}} & \boldsymbol{R}_{r_{1}}^{1 / 2} \boldsymbol{h}_{w_{1}} \cdots \boldsymbol{R}_{r_{M_{t}-1}}^{1 / 2} \boldsymbol{h}_{w_{M_{t}-1}}\end{array}\right] \boldsymbol{P}$, where $\boldsymbol{h}_{w_{i}}=\left[h_{w_{i_{0}}}, h_{w_{i_{1}}}, \ldots, h_{w_{i_{M_{r}-1}}}\right]^{T}$ represents the fast fading channel from the $i$-th transmit antenna to the correlated receiver array. Note that the different transmit antennas experience different correlation matrices upon reception. The reason is that the signals from different base stations may see a very different angular spread at the terminal. Interestingly, this leads to a practical instance of the non-Kronecker correlation structure, evoked recently in [22].

The precoder $\boldsymbol{F}$ is optimized by the transmitters, with access only to long-term statistical channel state information, given by the path loss and correlation matrices, $\mathbf{P}$ and $\boldsymbol{R}$. The receiver is assumed to know $\boldsymbol{H}$ perfectly. This is consistent with a practical multicell signaling overhead.

We let $B=M_{t}$ and have that, for all generalized complex orthogonal designs, $\boldsymbol{C}(\boldsymbol{x}) \boldsymbol{C}^{H}(\boldsymbol{x})=a \sum_{k=0}^{K-1}\left|x_{k}\right|^{2} \boldsymbol{I}_{M_{t}}$, where
$\boldsymbol{I}_{M_{t}}$ is the $M_{t}$-sized identity matrix and $a$ depends on the chosen orthogonal design [4]. Now, define the scalar $\alpha \triangleq$ $\|\boldsymbol{H} \boldsymbol{F}\|_{F}^{2}$, where $\|\cdot\|_{F}$ is the Frobenius norm. Using general results from [21], for our special case, we know that this OSTBC system with precoder $\boldsymbol{F}$ and channel correlation matrix $\boldsymbol{R}$ has an equivalent Single-Input Single-Output (SISO) formulation, where all input symbols $x_{k}$ experience the same channel gain $\sqrt{\alpha}$ and independent noise $v_{k}^{\prime} \sim \mathcal{C N}\left(0, \sigma_{v}^{2} / a\right)$, giving the output $y_{k}^{\prime}=\sqrt{\alpha} x_{k}+v_{k}^{\prime}, k \in\{0,1, \ldots, K-1\}$.

\section{Minimum SER PRECOding For CoOperative DIVERSITY}

From the above SISO formulation, the instantaneous received $\operatorname{SNR} \gamma$ is expressed as

$$
\gamma \triangleq\left(a \sigma_{x}^{2} \alpha\right) / \sigma_{v}^{2}=\delta \alpha=\delta\|\boldsymbol{H} \boldsymbol{F}\|_{F}^{2}, \quad \delta=\left(a \sigma_{x}^{2}\right) / \sigma_{v}^{2} .
$$

When all the symbols go through the same channel, the average SER of the system is [21]

$\mathrm{SER} \triangleq \operatorname{Pr}\{$ Error $\}=\int_{0}^{\infty} \operatorname{Pr}\{$ Error $\mid \gamma\} p_{\gamma}(\gamma) d \gamma=\int_{0}^{\infty} \operatorname{SER}_{\gamma} p_{\gamma}(\gamma) d \gamma$

where $p_{\gamma}(\gamma)$ is the probability density function (pdf) of SNR $\gamma$, while $\operatorname{SER}_{\gamma}$ is the symbol error probability for a given $\gamma$ and bit-to-symbol mapping (e.g. $M$-PSK).

We want to find $\boldsymbol{F}$ such that the exact SER is minimized, under a sum power constraint on the transmit block $Z \triangleq$ $\boldsymbol{F} \boldsymbol{C}(\boldsymbol{x})$. Such a constraint makes engineering sense when we assume many symmetrically distributed and mobile users. This is equivalent to an optimized power distribution across the cell, where each user is allocated the same multicell total power.

With the total power available $P$, the pooled power constraint across all $M_{t}$ antennas is $a K \sigma_{x}^{2} \operatorname{Tr}\left\{\boldsymbol{F} \boldsymbol{F}^{H}\right\}=P$. The problem of minimizing the SER can then be expressed as:

Problem 1:

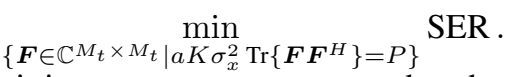

The transmitting antennas are assumed to be uncorrelated (unlike the receive antennas), and by applying Theorem 1 of [21], we show that the minimum SER precoder $\boldsymbol{F}$ is limited to the family of diagonal precoders, with real and non-negative diagonal elements. Note that the authors of that paper did not consider different path loss specifically, but we observe that by building scaled correlation matrices $\boldsymbol{R}_{r_{i}}^{\prime}=\rho_{i} \boldsymbol{R}_{r_{i}}$, their theorem can be made to hold here as well.

The problem of optimal precoding for cooperative diversity is thus reduced to an optimal power allocation (PA) over $M_{t}$ transmit antennas, and implementing this precoder is fully compatible with distributed processing in each cell (rather than multicell joint processing). For the PA optimization, we begin with the SER expression, and then resort to a simpler criterion.

\section{A. Optimal SER Precoder}

It is well-known that a simple, closed-form expression of the exact SER in (3) is not obtainable. Also, minimization of the SER with respect to the power values $f_{i}^{2}$ is difficult, as direct differentiation gives expressions of an order proportional to the number of antennas $M_{t} M_{r}$. This makes finding the optimal 


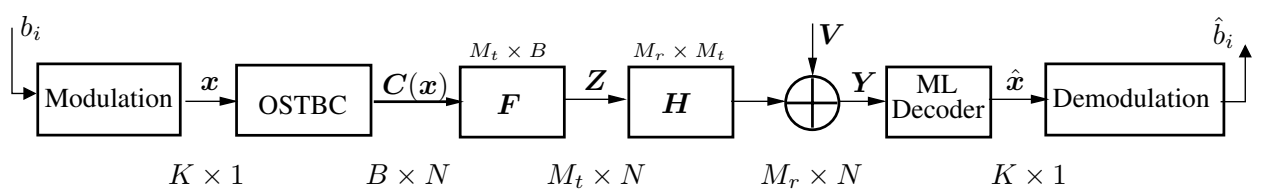

Fig. 1. Block model of a linearly precoded MIMO-system.

PA complicated, but the task is feasible with the iterative algorithm of [21].

In general, the optimal PA depends on the receive correlation conditions, the path loss $\rho_{i}$ and the average SNR. As a special case, if the SNR-related coefficient $\delta=a \sigma_{x}^{2} / \sigma_{v}^{2} \rightarrow \infty$, with non-zero path-loss and eigenvalues of $\boldsymbol{R}_{i}$, then we can show, using the argumentation of [23], that the optimal PA will approach the even distribution $f_{i}^{2}=1 / M_{t}$. We now approach the optimization via a different optimality criterion.

\section{Reduced Complexity Approaches to Precoder OPTIMIZATION}

\section{A. Closed-Form Precoder for Equal Average SNR}

Now, assume that slow power control is activated at the BSs, so the path loss coefficients are equal, while the correlation matrices remain possibly different. We generalize the equal diversity spread principle, introduced in [21], to the case of $M_{t} \geq 2$ transmit antennas, and formulate a closed-form precoder. For efficient notation, define the set $\mathcal{S}=\left\{0,1, \ldots, M_{t}-1\right\}$.

1) The Equal Diversity Spread Principle: Given $\boldsymbol{F}$ diagonal and normalize as $P /\left(a K \sigma_{x}^{2}\right)=1$, we have $\operatorname{Tr}\left\{\boldsymbol{F} \boldsymbol{F}^{H}\right\}=$ $\sum_{i=0}^{M_{t}-1} f_{i}^{2}=1$. The receive correlation matrices $\boldsymbol{R}_{r_{i}}$ of (1), have eigen-decomposition $\boldsymbol{R}_{r_{i}}=\boldsymbol{V}_{r_{i}} \boldsymbol{\Lambda}_{r_{i}} \boldsymbol{V}_{r_{i}}^{H}$. The instantaneous SNR writes as $\gamma=\delta \sum_{i=0}^{M_{t}-1} \rho_{i} f_{i}^{2}\left\|\boldsymbol{h}_{c_{i}}\right\|^{2}=$ $\delta \sum_{i=0}^{M_{t}-1} \sum_{j=0}^{M_{r}-1} \rho_{i} f_{i}^{2} \lambda_{i_{j}}\left|h_{w_{i_{j}}}^{\prime}\right|^{2}$, where $h_{w_{i_{j}}}^{\prime}$ is the $j$-th element of $\boldsymbol{h}_{w_{i}}^{\prime}=\boldsymbol{V}_{r_{i}}^{H} \boldsymbol{h}_{w_{i}}$ and $\lambda_{i_{j}}$ is the $j$-th eigenvalue of $\boldsymbol{R}_{r_{i}}$. The matrices $\boldsymbol{V}_{r_{i}}$ are unitary, so the elements of $\boldsymbol{h}_{w_{i}}^{\prime}$ follow the same distribution as those of $\boldsymbol{h}_{w_{i}}$. Thus, the instantaneous SNR of (2) is a random variable on the form $\gamma=\sum_{l=0}^{N-1} a_{l}\left|Z_{l}\right|^{2}$, with deterministic $a_{l}$ and random $Z_{l} \sim \mathcal{C N}(0,1)$.

Equal path loss coefficients, $\rho_{i}=1, i \in \mathcal{S}$, yields the SNR-expression $\gamma=\delta \sum_{i=0}^{M_{t}-1} f_{i}^{2}\left(\sum_{j=0}^{M_{r}-1} \lambda_{i_{j}}\left|h_{w_{j_{i}}}^{\prime}\right|^{2}\right)$, a simplified sum of $M_{t} M_{r}$ uncorrelated, weighted, diversity branches. In accordance with the proposed equal diversity spread principle [21], we attempt to spread the energy evenly across all these branches, making the weights as equal as possible under a sum power constraint. We use $\operatorname{Tr}\left\{\boldsymbol{R}_{r_{i}}\right\}=$ $M_{r}, i \in \mathcal{S}$, and find that the arithmetic mean of the weights is $m=1 /\left(M_{t} M_{r}\right) \sum_{i=0}^{M_{t}-1} \sum_{j=0}^{M_{r}-1} f_{i}^{2} \lambda_{i_{j}}=\frac{1}{M_{t}}$.

We propose that the weights be decided according to the following minimum variance problem:

Problem 2:

$$
\min _{\left\{f_{i} \geq 0, i \in \mathcal{S} \mid \sum_{i=0}^{M_{t}-1} f_{i}^{2}=1\right\}} \sum_{i=0}^{M_{t}-1} \sum_{j=0}^{M_{r}-1}\left(f_{i}^{2} \lambda_{i_{j}}-\frac{\delta}{M_{t}}\right)^{2} .
$$

The simple, closed-form solution is stated in Theorem 1 .
Theorem 1: Each power weight $f_{i}, i \in \mathcal{S}$, is found as

$$
f_{i}=\sqrt{\frac{\beta}{\sum_{j=0}^{M_{r}-1} \lambda_{i_{j}}^{2}}}, \text { where } \beta=\frac{1}{\sum_{i=0}^{M_{t}-1} \frac{1}{\sum_{j=0}^{M_{r}-1} \lambda_{i_{j}}^{2}}}
$$

Proof: We define $g=\sum_{i=0}^{M_{t}-1} \sum_{j=0}^{M_{r}-1}\left(f_{i}^{2} \lambda_{i_{j}}-\frac{\delta}{M_{t}}\right)^{2}+$ $\beta^{\prime}\left(\sum_{i=0}^{M_{t}-1} f_{i}^{2}-1\right)$, using a Lagrangian multiplier $\beta^{\prime}$ with constraint $\sum_{i=0}^{M_{t}-1} f_{i}^{2}=1$. Differentiation wrt the transmit powers $f_{i}^{2}$ yields $\frac{\delta g}{\delta f_{i}^{2}}=\sum_{j=0}^{M_{r}-1} 2\left(f_{i}^{2} \lambda_{i_{j}}-\frac{\delta}{M_{t}}\right) \lambda_{i_{j}}+\beta^{\prime}=$ $2 f_{i}^{2} \sum_{j=0}^{M_{r}-1} \lambda_{i_{j}}^{2}-\frac{2 \delta M_{r}}{M_{t}}+\beta^{\prime}=0$. Using $\beta=\frac{\delta M_{r}}{M_{t}}-\frac{\beta^{\prime}}{2}$, yields $f_{i}=\sqrt{\frac{\beta}{\sum_{j=0}^{M_{r}-1} \lambda_{i_{j}}^{2}}}, i \in \mathcal{S}$, where $\beta=\frac{1}{\sum_{i=0}^{M_{t}-1} \frac{1}{\sum_{j=0}^{M_{r}-1} \lambda_{i_{j}}^{2}}}$ comes from the sum power constraint.

In words, the power is distributed so that a transmit antenna $i$, for which the receive correlation is negligible, will be allocated more power than an antenna $j$ that sees a higher level of correlation at the receiver. In the first case, the correlation matrix $\boldsymbol{R}_{r_{i}}$ is close to identity, s.t. $\sum_{j=0}^{M_{r}-1} \lambda_{i_{j}}^{2} \sim M_{r}$, while in the second case $\boldsymbol{R}_{r_{j}}$ has more different eigenvalues, so the sum of their squares increases compared to the uncorrelated case. Antennas that see similar receive correlation conditions will have the same amount of power allocated for transmission.

From Theorem 1, note that the PA after the equal diversity spread principle is SNR independent, unlike the exact SER (3). It turns out this is a good criterion for low to moderate values of SNR and shows sub-optimality at high SNR.

2) A Gaussian Approximation: Next, we pursue another approach, not presented in [21], which also yields the above presented idea of equal diversity spread. As $\gamma$ is a sum of $M_{t} M_{r}$ equally weighted, independent random variables, the central limit theorem and the known positivity of $\gamma$ motivates approximating its distribution by a truncated Gaussian. For $\gamma \geq 0$, we define the pdf

$p_{\gamma}(\gamma)=\frac{C}{\sigma_{\gamma} \sqrt{2 \pi}} e^{-\frac{\left(\gamma-m_{\gamma}\right)^{2}}{2 \sigma_{\gamma}^{2}}}, \frac{1}{C}=\int_{0}^{\infty} \frac{1}{\sigma_{\gamma} \sqrt{2 \pi}} e^{-\frac{\left(\gamma^{\prime}-m_{\gamma}\right)^{2}}{2 \sigma_{\gamma}^{2}}} d \gamma^{\prime}$,

The correction factor $C$ ensures $\int_{0}^{\infty} p_{\gamma}(\gamma) \delta \gamma=1$. With $M$-PSK mapping, the $\mathrm{SER}_{\gamma}$ of (3) is $\operatorname{SER}_{\gamma}=$ $\frac{1}{\pi} \int_{0}^{\frac{(M-1) \pi}{M}} e^{-\frac{g \text { PSK }}{\sin ^{2}(\theta)} \gamma} d \theta$ [21]. Using the approximated pdf, yields the SER

$$
\begin{aligned}
\mathrm{SER} & \approx C \int_{0}^{\infty} \operatorname{SER}_{\gamma} \frac{1}{\sigma_{\gamma} \sqrt{2 \pi}} e^{-\frac{\left(\gamma-m_{\gamma}\right)^{2}}{2 \sigma_{\gamma}^{2}}} d \gamma \\
& =\frac{C}{\sigma_{\gamma} \pi \sqrt{2 \pi}} \int_{0}^{\frac{(M-1) \pi}{M}} \int_{0}^{\infty} e^{-\frac{\left(\gamma-m_{\gamma}\right)^{2}}{2 \sigma_{\gamma}^{2}} e^{-\frac{g_{\mathrm{PSK}}}{\sin ^{2}(\theta)} \gamma} d \gamma d \theta .}
\end{aligned}
$$

We substitute $s=-g_{\mathrm{PSK}} / \sin ^{2}(\theta)$ in (8), and use the correction factor $C$, to approximate the Gaussian $\operatorname{mgf} \phi_{\gamma}(s)$ [24], 
despite the truncation. From this, we rewrite the SER

$$
\mathrm{SER} \approx \frac{1}{\pi} \int_{0}^{\frac{(M-1) \pi}{M}} \exp \left(m_{\gamma} s+\frac{1}{2} \sigma_{\gamma}^{2} s^{2}\right) d \theta .
$$

Now, we propose to minimize the integrand of (9), for any value of $\theta$. Then, the problem reduces to finding the minimum of $\left(m_{\gamma}+\frac{1}{2} \sigma_{\gamma}^{2} s\right)$. The mean and variance of $\gamma$ are derived in a straightforward manner, and given as $m_{\gamma}=\delta M_{r}$ and $\sigma_{\gamma}^{2}=$ $\delta^{2} \sum_{i=0}^{M_{t}-1}\left(f_{i}^{2}\right)^{2} \sum_{j=0}^{M_{r}-1} \lambda_{i_{j}}^{2}$, respectively. Thus, the problem of minimizing the SER is further reduced to minimizing the variance $\sigma_{\gamma}^{2}$, and the solution is found using the same approach as for Theorem 1 , giving $f_{i}, i \in \mathcal{S}$, as

$$
\begin{gathered}
f_{i}=\sqrt{\frac{\beta}{\sum_{j=0}^{M_{r}-1} \lambda_{i_{j}}^{2}}}, \text { where } \\
\sum_{i=0}^{M_{t}-1} f_{i}^{2}=1 \Longrightarrow \beta=\frac{1}{\sum_{i=0}^{M_{t}-1} \frac{1}{\sum_{j=0}^{M_{r}-1} \lambda_{i_{j}}^{2}}} .
\end{gathered}
$$

That this closed form precoder is identical to the one obtained from the equal diversity spread principle, is interesting and gives some insights into the justification of the use of this principle, beyond the simple intuition.

\section{B. Precoder for Unequal Average SNR}

In the most general case, the different transmit antennas may see a different average path loss. If the path losses are substantially different and slow power control is not activated, the Gaussian approximation above loses credibility. Here, we propose to optimize a version of the PEP-based metric of [25], modified to accommodate the different path losses $\rho_{i}, i \in \mathcal{S}$.

$$
\max _{\left\{\boldsymbol{F} \in \mathbb{C}^{M_{t} \times B}\right\}} \operatorname{det}\left(\boldsymbol{I}_{M_{t} M_{r}}+\frac{a d_{\min }^{2}}{4 \sigma_{v}^{2}} \boldsymbol{R}_{\boldsymbol{P}^{\frac{1}{2}}}\left[\left(\boldsymbol{F}^{*} \boldsymbol{F}^{T}\right) \otimes \boldsymbol{I}_{M_{r}}\right] \boldsymbol{R}_{\boldsymbol{P}^{\frac{1}{2}}}\right),
$$

for $\operatorname{Tr}\left\{\boldsymbol{F} \boldsymbol{F}^{H}\right\}=1$, where $d_{\text {min }}^{2}$ is the minimum intersymbol distance for the modulation scheme, while $\boldsymbol{R}_{\boldsymbol{P}}=$ $\operatorname{diag}\left(\left[\rho_{0} \mathbf{1}_{1 \times M_{r}}, \quad \rho_{1} \mathbf{1}_{1 \times M_{r}}, \ldots \rho_{M_{t}-1} \mathbf{1}_{1 \times M_{r}}\right]\right) \boldsymbol{R}$ contains correlation and path loss. With uncorrelated transmitters, it still holds that the optimal $\boldsymbol{F}$ can be chosen as diagonal, real and non-negative, and we may, as in [25], simplify the optimization. Taking the logarithm, and using $\sum_{i=0}^{M_{t}-1} f_{i}^{2}=1$, and $k=a d_{\min }^{2} /\left(4 \sigma_{v}^{2}\right)$, yields the equivalent problem

$$
\max _{\left\{\boldsymbol{F}=\operatorname{diag}\left(\left[f_{0}, \ldots, f_{M_{t}-1}\right]\right), f_{i} \in \mathbb{R}_{+}\right\}} \sum_{i=0}^{M_{t}-1} \sum_{j=0}^{M_{r}-1} \log \left(1+k \rho_{i} f_{i}^{2} \lambda_{i_{j}}\right) .
$$

Interestingly, the maximization of the above sum of logarithms resembles the well-known capacity maximization problem, for which the solution is the classical water-filling. Unfortunately, because each power variable $f_{i}^{2}$ appears in $M_{r}$ terms of the sum, it is a totally different problem, except in special cases. Note that the function $\sum_{i=0}^{M_{t}-1} \sum_{j=0}^{M_{r}-1} \log (1+$ $\left.k \rho_{i} f_{i}^{2} \lambda_{i_{j}}\right)$ in (12) is concave, and the partial double derivative $\partial^{2} /\left(\partial f_{k}^{2}\right)^{2}, k \in \mathcal{S}$ is always negative or zero.
1) Solution for Uncorrelated Case: As a special case, we assume that both sides of the channel are uncorrelated, so that $\boldsymbol{R}_{r_{i}}=\boldsymbol{I}_{M_{r}} \forall i$, and the problem in (12) simplifies to

$$
\max _{\left\{\boldsymbol{F} \in \operatorname{diag}\left(\mathbb{R}^{+}\right)^{M_{t} \times 1}\right\}} M_{r} \sum_{i=0}^{M_{t}-1} \log \left(1+k \rho_{i} f_{i}^{2}\right), \quad \sum_{i=0}^{M_{t}-1} f_{i}^{2}=1 .
$$

We recognize this problem as one for which the solution is the classical water-filling, finding $f_{i}, i \in \mathcal{S}$, such that

$$
f_{i}^{2}=\left(\mu-\frac{1}{k \rho_{i}}\right)_{+}, \quad(\cdot)_{+}=\max (\cdot, 0), \sum_{i=0}^{M_{t}-1} f_{i}^{2}=1 .
$$

This special setting of unequal average SNR and no correlation, can be seen as an equivalent to the optimization problem presented in [26]. There, the authors assume transmit correlation, no receive correlation and equal average SNR. These two scenarios may be compared by substituting the transmit correlation matrix $\boldsymbol{R}_{a, T}^{1 / 2}$ in [26] with our diagonal path loss matrix $\boldsymbol{P}$, a simplification. Following the steps in [26] then leads to the same water-filling solution as in (14), thus the two approaches correspond.

2) Modified Water-filling Solution for Correlated Case: We return to the most general case where each transmitter experiences a different path loss and receive correlation. To find a solution to the optimization in (12), we introduce a Lagrange multiplier $\mu$, with power constraint $\operatorname{Tr}\left\{\boldsymbol{F} \boldsymbol{F}^{H}\right\}=1$, and differentiate with respect to $f_{i}^{2}$. This yields $M_{t}$ equations on the form

$$
\sum_{j=0}^{M_{r}-1} \frac{k \rho_{i} \lambda_{i_{j}}}{1+k \rho_{i} f_{i}^{2} \lambda_{i_{j}}}+\mu=0, \quad \text { for } i \in \mathcal{S} .
$$

For each equation, we optimize with respect to the first term in the sum, fixing the other $M_{r}-1$ terms in a constant $c_{i}=\sum_{j=1}^{M_{r}-1} \frac{k \rho_{i} \lambda_{i}}{1+k \rho_{i} f_{i}^{2} \lambda_{i_{j}}}$. The eigenvalues are assumed to be ordered, so $\lambda_{i_{0}} \geq \lambda_{i_{j}}, \forall j \neq 0$. Now, (15) can be rewritten as

$$
\frac{k \rho_{i} \lambda_{i_{0}}}{1+k \rho_{i} f_{i}^{2} \lambda_{i_{0}}}=-\left(\mu+c_{i}\right), \quad \text { for } i \in \mathcal{S} \text {. }
$$

We impose the power constraint $\sum_{i=0}^{M_{t}-1} f_{i}^{2}=1$, and attempt to find $\mu$. This approach means solving an equation of degree $M_{t}$, so, if a closed-form solution is desired, it holds for $M_{t} \leq 4$.

$$
\sum_{i=0}^{M_{t}-1} f_{i}^{2}=-\sum_{i=0}^{M_{t}-1}\left(\frac{1}{\mu+c_{i}}+\frac{1}{k \rho_{i} \lambda_{i_{0}}}\right)=1 .
$$

Of the possible values for $\mu$, we use the one that minimizes $\sum_{i=0}^{M_{t}-1}\left|f_{i}^{2}\right|$, and update the powers $f_{i}^{2}=-\frac{1}{\mu+c_{i}}-\frac{1}{k \rho_{i} \lambda_{i_{0}}}$, for $i \in \mathcal{S}$. This procedure is repeated until convergence is reached, although no formal proof for the conditions of convergence are available yet.

3) Modified Fixed-Point Iteration: Depending on the path loss, the SNR and receive correlation conditions, the above fixed-point iteration might not converge.

Assume the whole procedure of iteration $k+1$ can be expressed by the iteration function $\phi\left(\left(f_{i}^{2}\right)^{(k)}\right)$, so that the relation between two consecutive power values $\left(f_{i}^{2}\right)^{(k)}$ and $\left(f_{i}^{2}\right)^{(k+1)}$ is expressed as $\left(f_{i}^{2}\right)^{(k+1)}=\phi\left(\left(f_{i}^{2}\right)^{(k)}\right), i \in$ 


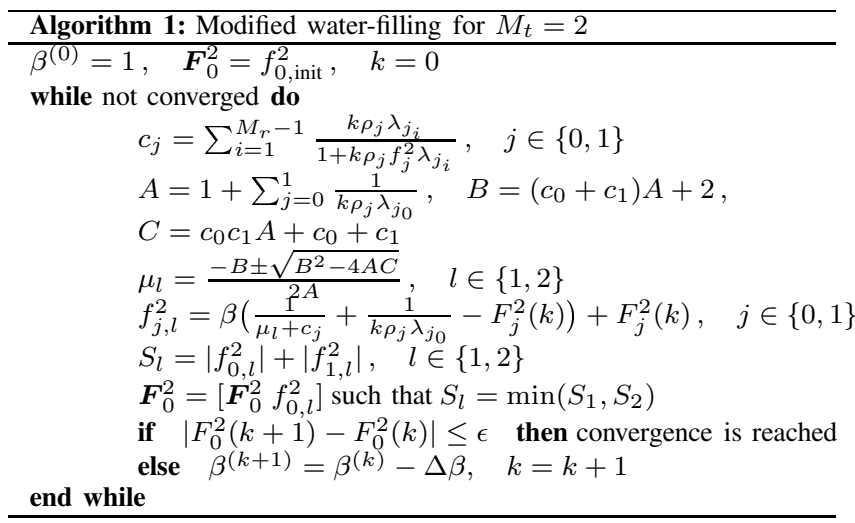

$\mathcal{S}$. From the theory on fixed-point iterations [27], nonconvergence may appear in cases where the derivative of the iteration function $\phi\left(\left(f_{i}^{2}\right)^{(k)}\right)$ is such that $\left|\phi^{\prime}\left(\left(f_{i}^{2}\right)^{(k)}\right)\right| \geq 1$. To avoid this, we slow down the change by solving an equivalent fixed-point problem: $\left(f_{i}^{2}\right)^{(k+1)}=\beta^{(k)} \phi\left(\left(f_{i}^{2}\right)^{(k)}\right)+$ $\left(1-\beta^{(k)}\right)\left(f_{i}^{2}\right)^{(k)}, 0<\beta^{(k)} \leq 1$. When convergence occurs, we have $\phi\left(\left(f_{i}^{2}\right)^{(k)}\right)=\left(f_{i}^{2}\right)^{(k)}$, so that $\left(f_{i}^{2}\right)^{(k+1)}=\left(f_{i}^{2}\right)^{(k)}$. The special case of $M_{t}=2$ is detailed in Algorithm 1.

If we can estimate the derivative of the iteration function, the coefficient $\beta$ may be directly initialized to a value that allows for convergence. Otherwise, we may set $\beta^{(0)}=1$ and update it for each iteration that has not lead to convergence, $\beta^{(k+1)}=\beta^{(k)}-\Delta \beta$, with $\Delta \beta>0$. This method is both more complex and more realistic than the minimum variance approach, taking into account the possibility of unequal path loss.

\section{Simulation And Results}

We present simulation results for some selected scenarios, including both equal and unequal average received SNR. All cases result in highly non-Kronecker correlation situations which prevent the use of previously published precoders for point-to-point space-time codes. In the case of unequal average SNR, we plot BER-results for the PEP-based modified waterfilling optimization of Section IV-B.3, and the precoding gain is evaluated through comparison with equal PA and also with the iterative approach to SER minimization of [21]. For the case of equal average SNR, we also include the equal diversity spread principle of Section IV-A.1.

Our chosen scenario has two single-antenna BSs, so $M_{t}=$ $M_{t_{0}}+M_{t_{1}}=2$, that transmit cooperatively to one MU with $M_{r}=4$ antennas. The Monte Carlo simulations are run over 2000 channel realizations. All simulations use receive correlation matrices $\boldsymbol{R}_{r_{0}}=\mathbf{1}_{M_{t}}$ and $\boldsymbol{R}_{r_{1}}=\boldsymbol{I}_{M_{t}}$, where $\mathbf{1}_{M_{t}}$ is an $M_{t} \times M_{t}$-sized matrix of all ones. The two cases correspond to a fully correlated link seen by one BS and a fully decorrelated one seen by the other BS, an extreme situation.

First, the equal average SNR case is simulated, for which the results are shown in Fig. 2. Next, given two cases of unequal SNR values, $\left[\rho_{0}, \rho_{1}\right]=[1,0.75]$ and $\left[\rho_{0}, \rho_{1}\right]=[0.75,1]$, we obtain what is shown in Fig. 3 and Fig. 4. The results show that the iterative PEP-minimizing algorithm gives the same performance as the proposed, low-complexity, equal diversitybased algorithm, for the equal SNR case, which motivates the

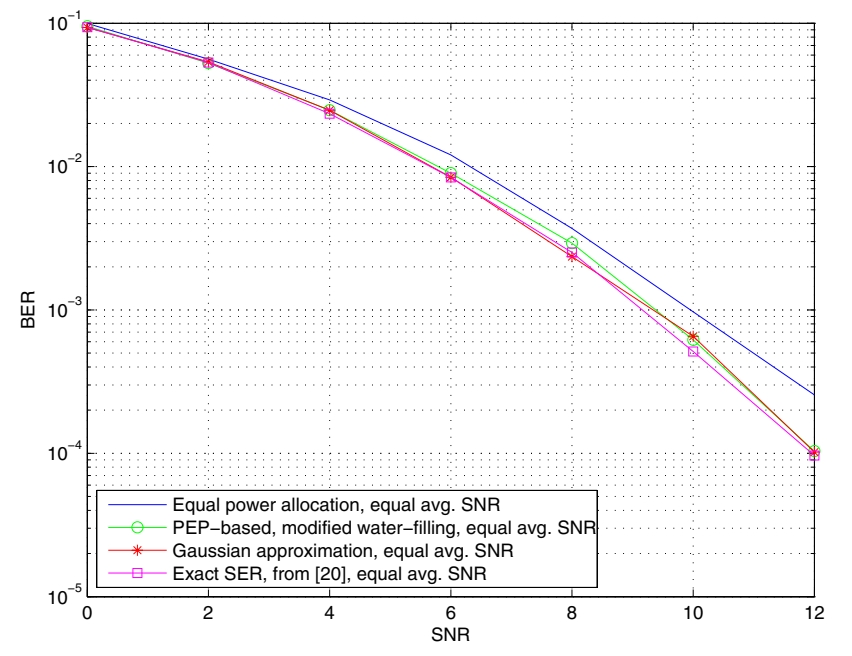

Fig. 2. Comparison of BER-performance versus SNR for $M_{t}=2, M_{r}=4$, in the case where $\boldsymbol{R}_{r_{0}}=\mathbf{1}_{M_{t}}$ and $\boldsymbol{R}_{r_{1}}=\boldsymbol{I}_{M_{t}}$ and for equal average SNR, $\left[\rho_{0}, \rho_{1}\right]=[1,1]$. The power allocations based on the Gaussian approximation of $\gamma$ and the PEP-measure have almost equal BER-results, outperforming the equal power allocation by close to $1 \mathrm{~dB}$ at BER $10^{-3}$.

use of the latter. In all cases, the methods presented loose only negligible performance to the SER optimal solution of [21].

For the case of unequal average SNR, we observe that the best BER results are obtained for the path loss $\left[\rho_{0}, \rho_{1}\right]=$ $[0.75,1]$, in Fig. 4. This is natural, as the second BS is the one that "sees" an uncorrelated receiver, which enables it to make the best use of the power.

\section{CONCLUSIONS}

We address the problem of distributed space-time coding for the cooperative downlink cellular network. The channel correlation structure is highly non-Kronecker because of the likely variations in local correlation properties from one cooperating device (e.g. BS) to the next. We compensate for this by using a distributed PA scheme.

Approaches to low-complexity precoder optimization are presented, including a closed-form precoder based on the equal diversity spread principle and iterative solutions based on modified water-filling. The maximum diversity principle is shown to be theoretically equivalent to a Gaussian assumption on the SNR obtained after combining all the diversity antennas.

\section{REFERENCES}

[1] J. Foschini, "Layered space-time architecture for wireless communications in a fading environment when using multiple antennas," Bell Labs Tech. J., vol. 1, no. 2, pp. 41-59, 1996.

[2] I. E. Telatar, "Capacity of multi-antenna Gaussian channels," European Trans. Telecommun., vol. 10, no. 6, pp. 585-595, Nov. 1999.

[3] D. Gesbert, M. Shafi, D. Shiu, and P. Smith, "From theory to practice: An overview of space-time coded MIMO wireless systems," IEEE $J$. Select. Areas Commun., vol. 21, no. 3, pp. 281-302, Apr. 2003.

[4] V. Tarokh, H. Jafarkhani, and A. R. Calderbank, "Space-time block codes from orthogonal designs," IEEE Trans. Inform. Theory, vol. 45, no. 5, pp. 1456-1467, July 1999.

[5] G. Jöngren, M. Skoglund, and B. Ottersten, "Combining beamforming and orthogonal space-time block coding," IEEE Trans. Inform. Theory, vol. 48, no. 3, pp. 611-627, Mar. 2002. 


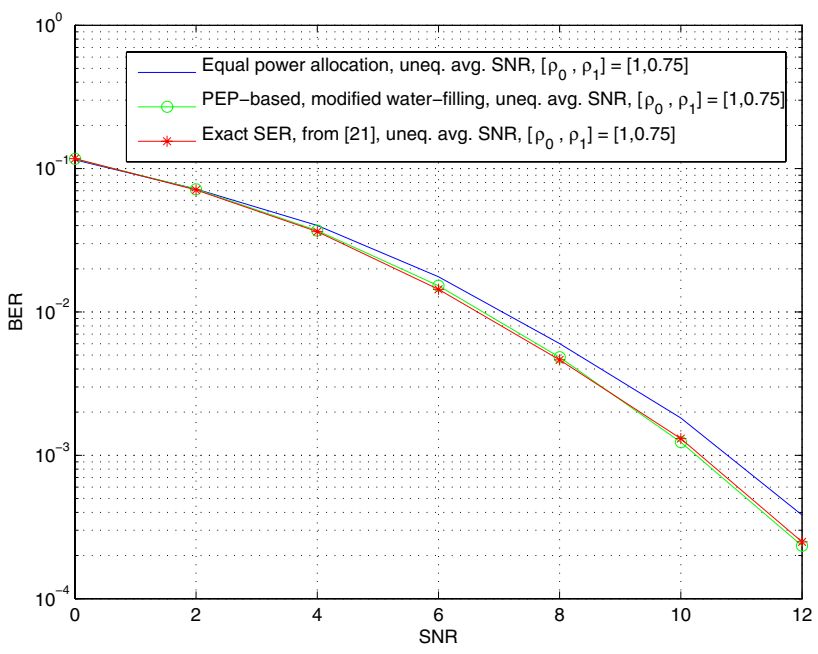

Fig. 3. Comparison of BER-performance versus SNR for $M_{t}=2, M_{r}=4$, in the case where $\boldsymbol{R}_{r_{0}}=\mathbf{1}_{M_{t}}$ and $\boldsymbol{R}_{r_{1}}=\boldsymbol{I}_{M_{t}}$ and for non-equal average SNR, realized by setting the path losses as $\left[\rho_{0}, \rho_{1}\right]=[1,0.75]$. The power allocation based on the PEP-measure outperforms the equal power allocation by close to $1 \mathrm{~dB}$ at BER $10^{-3}$.

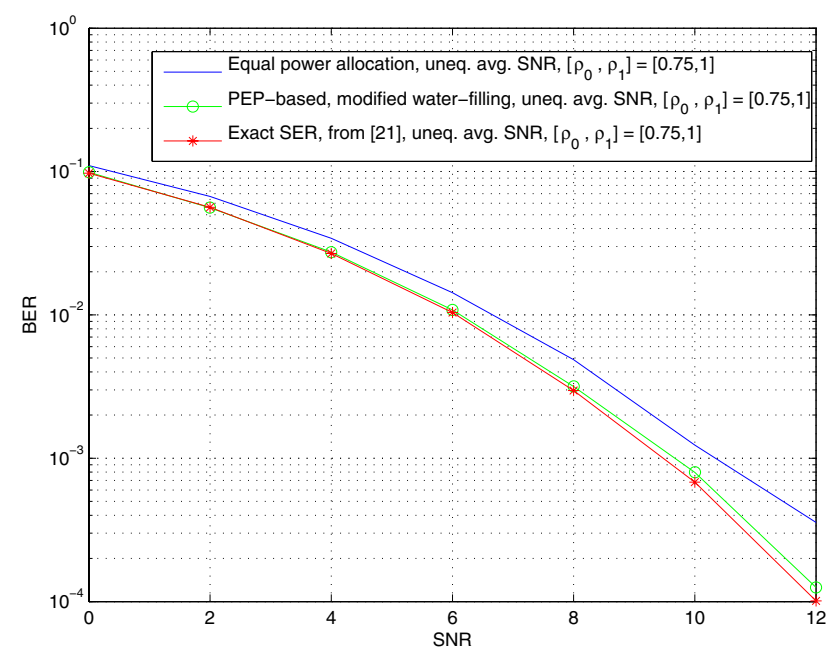

Fig. 4. Comparison of BER-performance versus SNR for $M_{t}=2, M_{r}=4$, in the case where $\boldsymbol{R}_{r_{0}}=\mathbf{1}_{M_{t}}$ and $\boldsymbol{R}_{r_{1}}=\boldsymbol{I}_{M_{t}}$ and for non-equal average SNR, realized by setting the path losses as $\left[\rho_{0}, \rho_{1}\right]=[0.75,1]$. The power allocation based on the PEP-measure outperforms the equal power allocation by close to $1 \mathrm{~dB}$ at BER $10^{-3}$.

[6] Y. Zhao, R. Adve, and T. J. Lim, "Precoding of orthogonal STBC with channel covariance feedback for minimum error probability," in Proc. IEEE Int. Conf. Indoor. Pers. Mobile Radio Commun., Sep. 2004, vol. 1, pp. 503-507.

[7] M. Vu and A. Paulraj, "Optimal linear precoders for mimo wireless correlated channelse with nonzero mean in space-time coded systems," IEEE Trans. Signal Processing, vol. 54, no. 6, pp. 2318-2332, June 2006.

[8] C. B. Peel, Q. H. Spencer, A. L. Swindlehurst, and M. Haardt, "An introduction to the multi-User MIMO downlink," IEEE Commun. Mag., pp. 60-67, Oct. 2004.

[9] S. Shamai and B. M. Zaidel, "Enhancing the cellular downlink capacity via co-processing at the transmitting end," in Proc. IEEE Semiannual Veh. Technol. Conf.-Spring, May 2001, vol. 3 pp. 1745-1749.

[10] H. Zhang, H. Dai, and Q. Zhou, "Base station cooperation for multiuser MIMO: Joint transmission and BS selection," in Proc. Conf. Inform. Sciences Syst., Mar. 2004.

[11] B. L. Ng, J. S. Evans, S. V. Hanly, and D. Aktas, "Transmit beamforming with cooperative base stations," in Proc. IEEE Int. Symp. Inform. Theory, Sep. 2005, pp. 1431-1435.

[12] A. Sendonaris, E. Erkip, and B. Aazhang, "User cooperation diversityPart I: System description. Part II: Implementation aspects and performance analysis," IEEE Trans. Commun., vol. 51, no. 11, pp. 1927-1948, Nov. 2003.

[13] J. N. Laneman and G. W. Wornell, "Distributed space-time-coded protocols for exploiting cooperative diversity in wireless networks," IEEE Trans. Inform. Theory, vol. 49, no. 10, pp. 2415-2425, Oct. 2003.

[14] T. Cover and A. El-Gamal, "Capacity theorems for the relay channel," IEEE Trans. Inform. Theory, vol. 25, no. 5, pp. 572-584, Sep. 1979.

[15] P. A. Anghel, G. Leus, and M. Kaveh, "Distributed space-time coding in cooperative networks," in Proc. Nordic Symp. Signal Processing, Oct. 2002.

[16] M. Janani, A. Hedayat, T. E. Hunter, and A. Nosratani, "Coded cooperation in wireless communications: Space-time transmission and iterative decoding," IEEE Trans. Signal Processing, vol. 52, no. 2, pp. 362-371, Feb. 2004.

[17] Y. Jing and B. Hassibi, "Distributed space-time coding in wireless relay networks," IEEE Trans. Wireless Commun., vol. 5, no. 12, pp. 35243536, Dec. 2006.

[18] S. Barbarossa, L. Pescosolido, D. Ludovici, L. Barbetta, and G. Scutari, "Cooperative wireless networks based on distributed space-time coding," in Proc. International Workshop Wireless Ad-Hoc Networks, June 2004, pp. 30-34.

[19] X. Li, "Space-time coded multi-transmission among distributed transmitters without perfect synchronization." IEEE Signal Processing Lett., vol. 11, no. 12, pp. 948-951, 2004.

[20] J. Mietzner and P. A. Hoeher, "On the duality of wireless systems with multiple cooperating transmitters and wireless systems with correlated antennas," in 14th IST Mobile Wireless Commun. Summit, June 2005.

[21] A. Hjørungnes and D. Gesbert, "Precoding of orthogonal space-time block codes in arbitrarily correlated MIMO channels: Iterative and closed-form solutions," IEEE Trans. Wireless Commun., vol. 6, no. 3, pp. 1072-1082, Mar. 2007.

[22] E. Bonek, H. Özcelik, M. Herdin, W. Weichselberger, and J. Wallace, "Deficiencies of a popular stochastic MIMO radio channel model," in Proc. 6th Int. Symp. Wireless Pers. Multimedia Commun., Oct. 2003.

[23] A. Hjørungnes and D. Gesbert, "Minimum exact SER precoder of orthogonal space-time block codes for correlated MIMO channels," Proc. IEEE GLOBECOM, Dec. 2004, vol. 1, pp. 111-115.

[24] K. Knight, Mathematical Statistics. New York: Chapman \& Hall/CRC, 2000.

[25] A. Hjørungnes, D. Gesbert, and J.Akhtar, "Precoding of space-time block coded signals for joint transmit-receive correlated MIMO channels," IEEE Trans. Wireless Commun., vol. 5, no. 3, pp. 492-497, Mar. 2006.

[26] H. Sampath and A. Paulraj, "Linear precoding for space-time coded systems with known fading correlations," IEEE Commun. Lett., vol. 6, no. 6, pp. 239-241, June 2002.

[27] A. Quarteroni, R. Sacco, and F. Saleri, Numerical Mathematics. New York: Springer Verlag, 2000. 